Proc. Estonian Acad. Sci. Biol. Ecol., 2002, 51, 1, 5-25

\title{
Commented list of macrophyte taxa of Lake Võrtsjärv
}

\begin{abstract}
Helle Mäemets
Võrtsjärv Limnological Station, Institute of Zoology and Botany, Estonian Agricultural University, 61101 Rannu, Tartumaa, Estonia; helle@zbi.ee

Received 14 May 2001, in revised form 3 October 2001

Abstract. A summary list of data on 114 taxa of macroflora, found by different researchers in L. Vorrtsjärv (area $270 \mathrm{~km}^{2}$ ) in 1912-2001, and a brief characterization of the habitats of the majority of the species and their frequency are presented. All these taxa are contained also in the floristic list of the largest lake of Estonia - L. Peipsi, connected with L. Võrtsjärv, which differs from that of L. Võrtsjärv mainly in having more rare species. Changes in the course of eutrophication are quite similar for both lakes. Expansion of reeds and decline of shallow-water and several submerged species have taken place.
\end{abstract}

Key words: changes during 20th century, parallelism with L. Peipsi, anthropogenic eutrophication.

\section{INTRODUCTION}

The list of the macrophytes of L. Võrtsjärv serves as a sequel to the commented floristic list of L. Peipsi (Mäemets \& Mäemets, 2000). It summarizes the data collected by all hydrobotanists who have studied the lake until the present time. It is interesting to compare the flora of L. Vorrtsjärv with that of other large lakes of the temperate zone and to bring out their common and specific features. Despite several methodological difficulties, the floristic lists with the estimates of abundance from different periods of the 20th century give an overview of changes that have occurred in the vegetation. The reasons for these changes are very complex. Besides current geological-geographical preconditions and the paludification of low shore areas, the state of the lake's vegetation reflects a simultaneous impact of several fluctuating factors: weather in the vegetation period, water level, drifting of sediments and plants (in large lakes). As these factors have long-term rhythms and are usually not covered with vegetation observations, investigators (e.g. Wallsten, 1981; Pokrovskaya et al., 1983; Coops \& Doef, 1996; Rintanen, 1996; Schmieder, 1998; etc.) have focused 
mainly on the process of anthropogenic eutrophication. Studies dealing with the impact of natural factors, for example the effect of water level on the vitality of the plants (Hultgren, 1988; Schmieder et al., in press), have been few. Anthropogenic eutrophication and acidification in drastic forms have affected water bodies only during the last 40-50 years. Although flax retting damaged several small lakes and lowering of water level as a result of land improvement caused a deterioration of several other lakes, macrophyte data from the first half of the 20th century, and even from the 1950s and 1960s, generally indicate a relatively undisturbed period for many Estonian lakes.

The present survey focuses on the comparison of the macroflora of L. Võrtsjärv and that of other large lakes of NE Europe, particularly L. Peipsi, as well as on changes that have taken place in the course of eutrophication. Despite the heterogeneity of the data at our disposal, their summarizing creates new ideas for future investigations. In regard to some groups of the biota, e.g. Chironomidae, the trophic level of L. Võrtsjärv in the 1960s was similar to that of less eutrophic L. Peipsi today (K. Kangur, pers. comm.). Thus, L. Võrtsjärv can possibly serve as a model of the future of L. Peipsi.

\section{GENERAL DESCRIPTION OF LAKE VÕRTSJÄRV}

The area of the second largest lake of Estonia (Fig. 1) is $270 \mathrm{~km}^{2}$, mean depth $2.8 \mathrm{~m}$, maximum depth $6.0 \mathrm{~m}$, the length along the north-south axis is $34.8 \mathrm{~km}$, and maximum width $14.8 \mathrm{~km}$. Among 18 inflows the Väike Emajõgi, Õhne, and Tänassilma rivers account for $70-75 \%$ of the total river discharge (Haberman et al., 1998). The outflow occurs via the Emajõgi River and Lake Peipsi $\left(3555 \mathrm{~km}^{2}\right)$. The Narva River connects the lake with the Gulf of Finland (Fig. 1). The water turnover time lasts nearly a year (Jaani, 1990). Lake Võrtsjärv is situated in the Central Estonian depression of preglacial origin and its bedrock is formed of Middle Devonian deposits, mainly sandstone. About $2 / 3$ of the lake bottom is covered with mud lying on marl, the latter is exposed only in some places (Fig. 2). The thickness of mud and marl increases towards the south (up to 7 and $4.7 \mathrm{~m}$, respectively). Varved clays lying under marl are exposed in the form of sandy, muddy-sandy, or clayey bottom mostly in the northern part of the lake. In places, underwater accumulations of boulders can be found (Veber, 1973). Although the lake is shallow, the range of water level fluctuations is large: annual mean $1.38 \mathrm{~m}$ and annual maximum $2.20 \mathrm{~m}$ (Jaani, 1973), the long-term mean of water level being $33.64 \mathrm{~m}$ a.s.l. (Järvet \& Nõges, 1998). Three parts (Fig. 3) can be distinguished in the lake: (a) the northern part with constant hydrochemical conditions; (b) the southernmost part with rich submerged and floating-leaved vegetation affected by the Väike Emajõgi River, and (c) the southern transition zone (Simm \& Starast, 1965).

The most noticeable long-term changes in the ionic composition of the water during the last 40 years have been increasing chloride and sulphate ion 


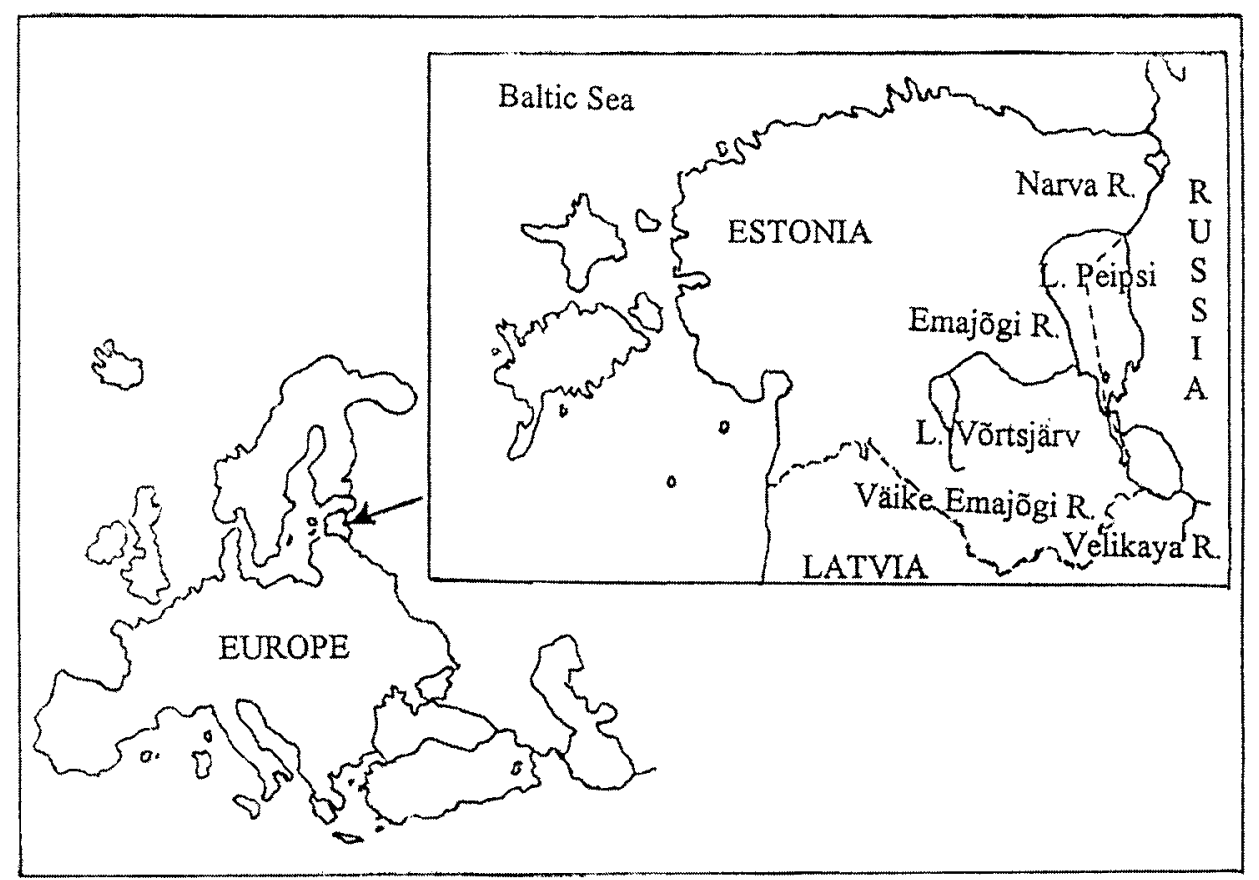

Fig. 1. Location of Lake Võrtsjärv.

concentrations. The means (over the period 1989-96) of these parameters are $10.9 \mathrm{mg} \mathrm{L}^{-1}$ for $\mathrm{Cl}^{-}$and $19.1 \mathrm{mg} \mathrm{L}^{-1}$ for $\mathrm{SO}_{4}^{-}$(Haberman et al., 1998). Also a decrease has occurred in the $\mathrm{Ca}^{2+} / \mathrm{Mg}^{2+}$ ratio. As the lake's buffering capacity is great, acidification does not present any serious danger (long-term mean $\mathrm{pH}$ 8.1). In comparison with the period 1962-66, the phosphate load increased 5.4 times and the nitrate load 1.4 times in the 1970s. However, the long-term data of the phosphate and nitrate concentrations (Fig. 4) in lake water do not show any consistent trend (Järvet \& Nõges, 1998). Since the beginning of measurements of total $\mathrm{P}$ and $\mathrm{N}$ in 1983, the concentration of total P has continuously decreased, while the concentration of total $\mathrm{N}$ has not undergone significant changes. The mean concentrations of total $\mathrm{P}$ and total $\mathrm{N}$ in the period 1983-96 (54 and $1600 \mu \mathrm{g} \mathrm{L}^{-1}$, respectively, Haberman et al., 1998), are characteristic of the eutrophic or hypertrophic stage. As the lake is shallow, the biological indices of its trophic state differ markedly for the periods of dry and rainy years. Thus overgrowing with macrophytes depends also on fluctuations in water level (Fig. 5). The mean Chl content for the period 1982-96 was $24.4 \mu \mathrm{g} \mathrm{L}^{-1}$, and $\mathrm{COD}_{\mathrm{Mn}}$ was $10.8 \mathrm{mg} \mathrm{O} \mathrm{L}^{-1}$ in 1968-96 (Haberman et al., 1998). Besides, heavy storms cause an intensive resuspension of sediment and a noteworthy increase in phosphorus content in water (Järvet \& Nõges, 1998). The mean water transparency in 1964-96 was $1.1 \mathrm{~m} \mathrm{SD}$. 


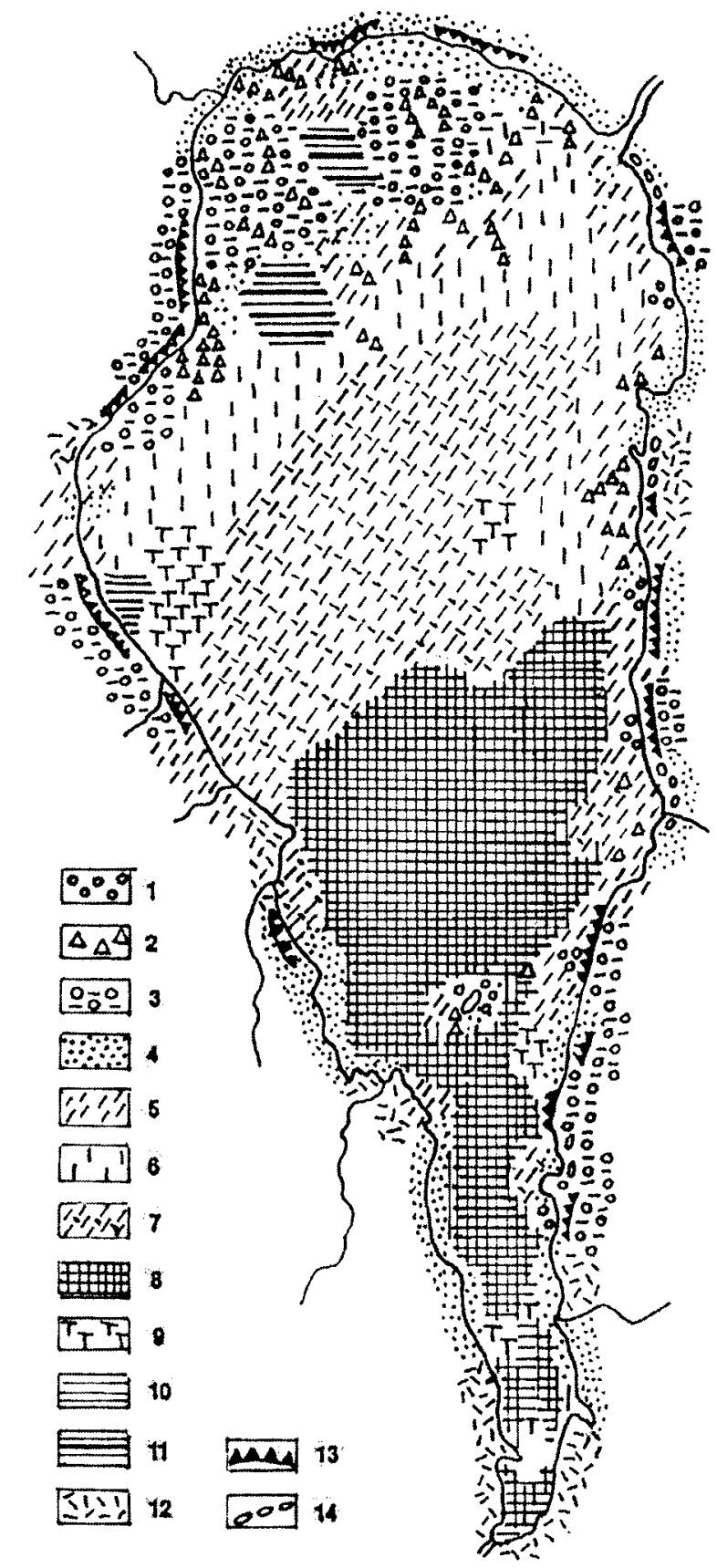

Fig. 2. Bottom sediments and shore types of Lake Võrtsjärv (after Raukas \& Mäemets, 1995): 1, Devonian sandstone; 2, boulders and stone heaps; 3, till; 4, varieties of sand; 5 , varieties of silt; 6 , silty pelite; 7 , sapropelic silty pelite; 8 , sapropel (often rich in mineral particles); 9 , marl; 10, clay; 11, varved clay; 12 , peaty shore; 13 , bluffs in different stages of development; 14, beach ridges. 


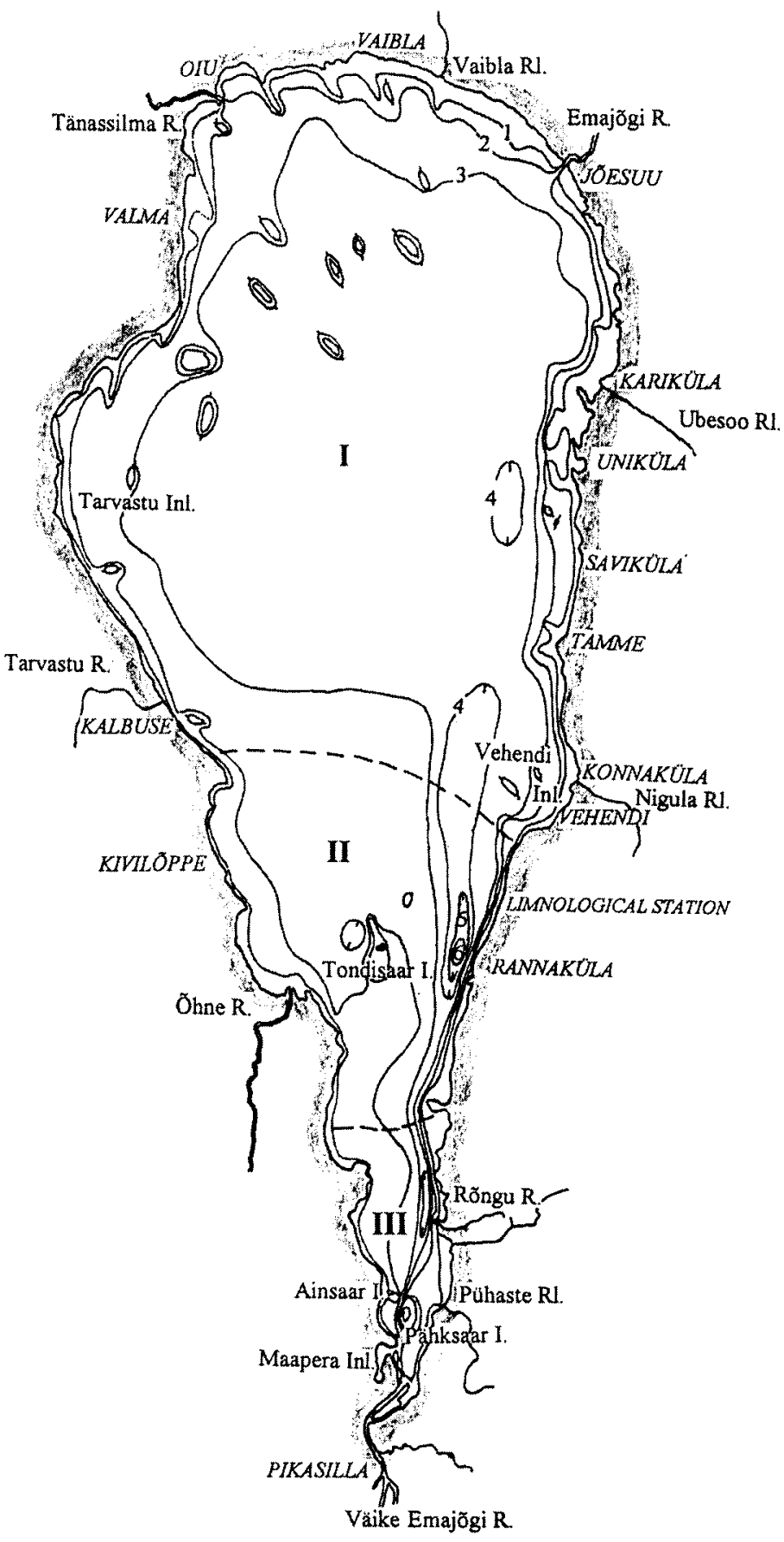

Fig. 3. Main hydrochemical zones of Lake Võrtsjärv (after Simm \& Starast, 1965): I - the northern part with stable hydrochemical conditions; II - the southern transition zone; III - the southernmost part with rich submerged and floating-leaved vegetation affected by the Väike Emajõgi River. Inl, inlet; I, island; Rl, rivulet; $\mathrm{R}$, river. 

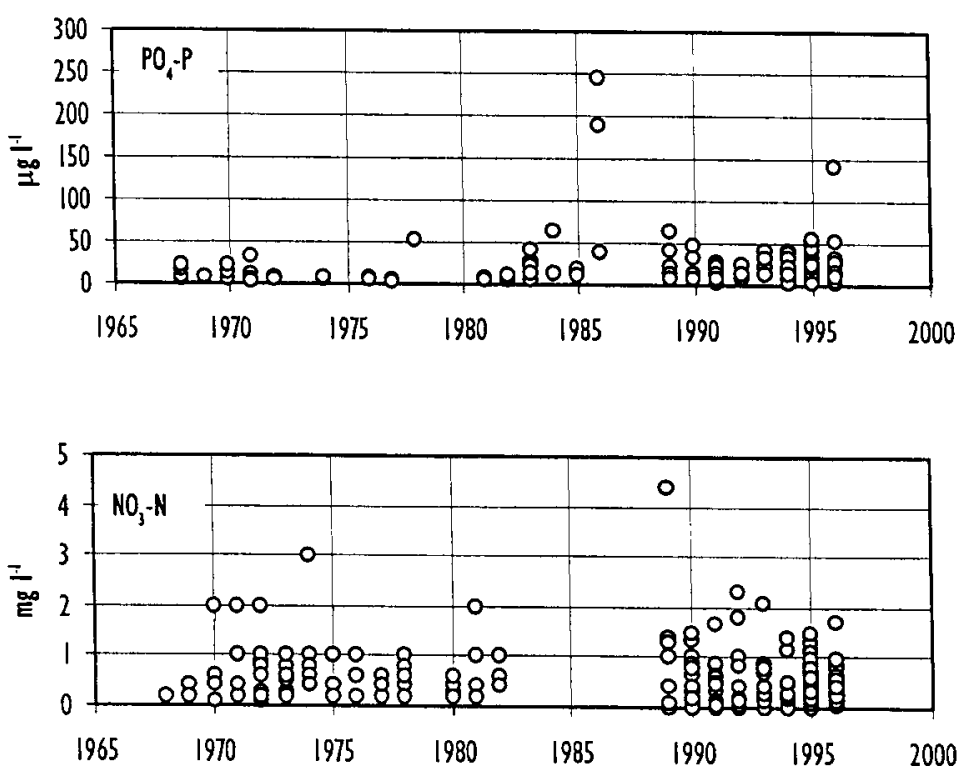

Fig. 4. Long-term data of phosphate and nitrate concentrations (Järvet \& Nõges, 1998).

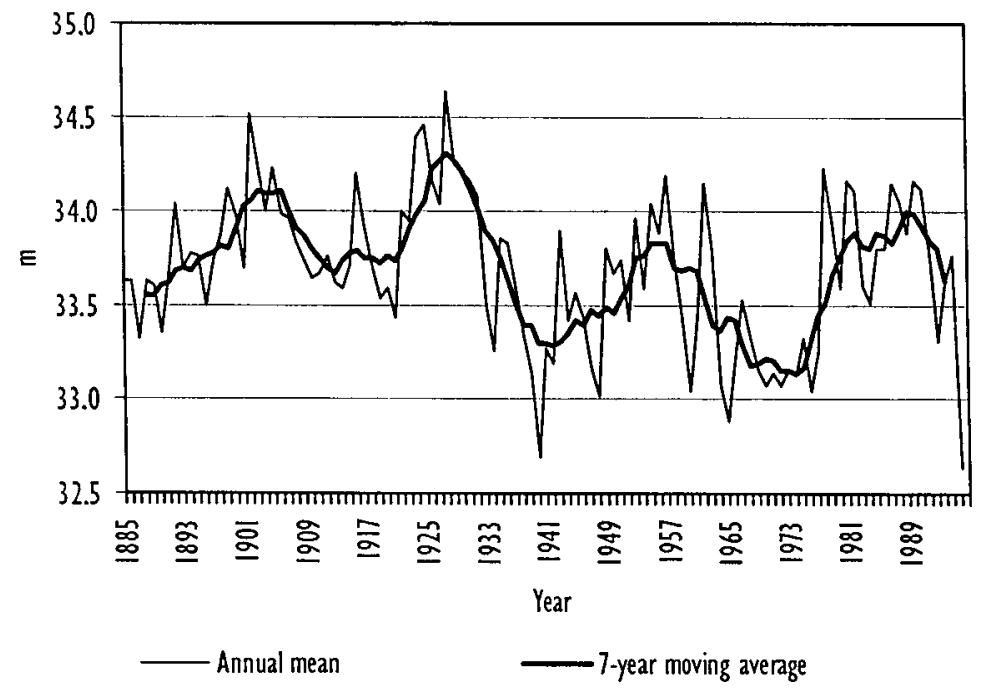

Fig. 5. Long-term fluctuation of the minimum water level of Lake Võrtsjärv (Järvet \& Nõges, 1998). 
Macrophytes covered $18.8 \%$ of the lake area in 1997 (Nõges et al., 1999) (Fig. 6). The maximum growth depth for submerged plants (Myriophyllum spicatum) in 1997 was $3.0 \mathrm{~m}$, for floating-leaved plants $1.8 \mathrm{~m}$, and for emergent plants $1.9 \mathrm{~m}$. At that time (in August 1997) the water level reached $33.30 \mathrm{~m}$ a.s.l., i.e. $34 \mathrm{~cm}$ less than the long-term mean (Feldmann, 2000).

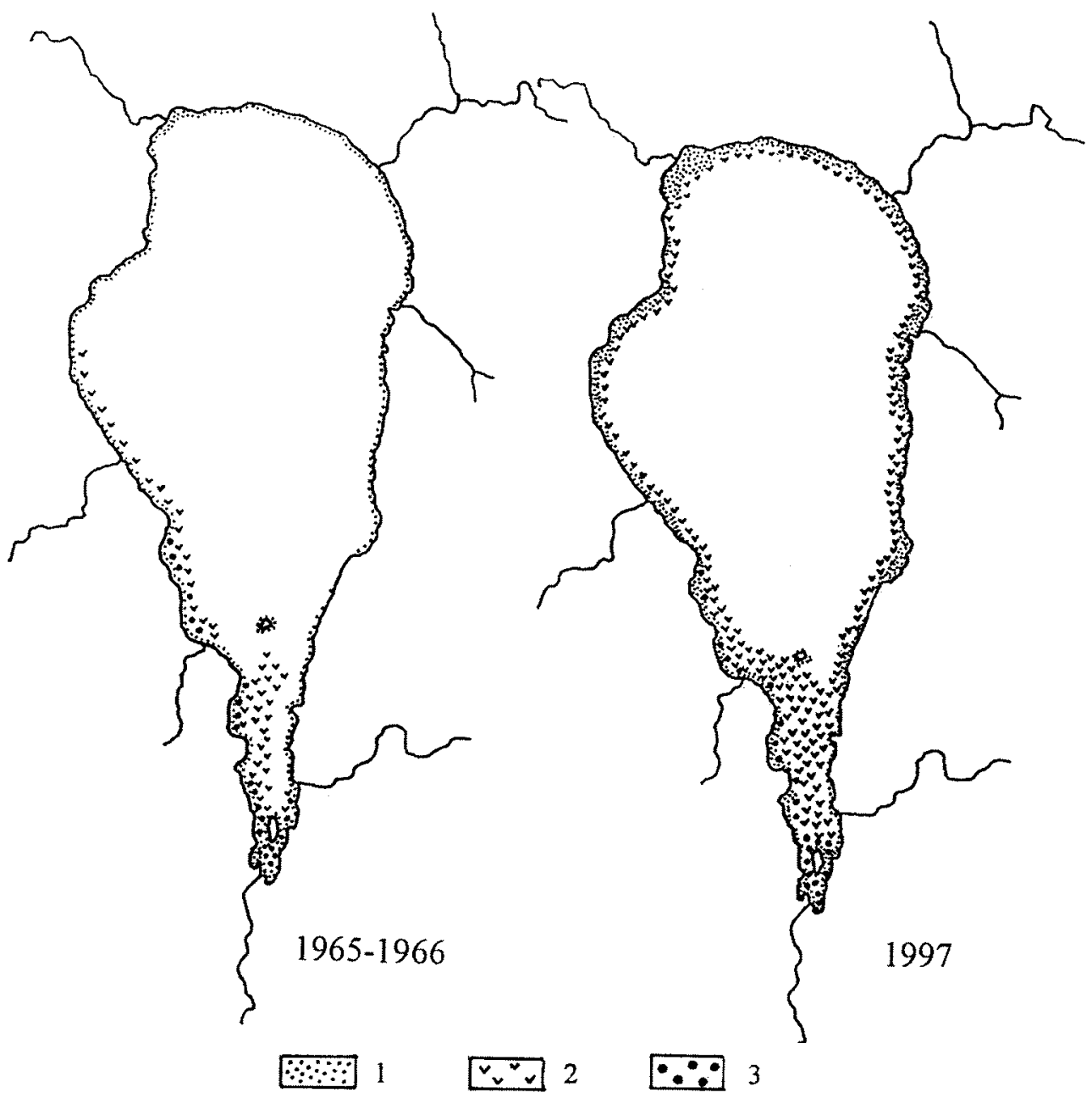

Fig. 6. The growth areas of dominating macrophyte species in Lake Võrtsjärv in 1965-66 (after Mäemets, 1973) and 1997 (after Feldmann, 2000): 1, Phragmites australis; 2, Myriophyllum spicatum; 3, Nuphar lutea. 


\section{MATERIAL AND METHODS}

The first observations of the vegetation of L. Võrtsjärv were made in the second half of the 19th century by Klinge, who formulated also the natural law stating that water bodies start to grow over from the leeward side (Klinge, 1890). The first survey of the lake's main vegetation regions as well as a floristic list (48 taxa) are presented in a treatise by Mühlen \& Schneider (1920), for which the material was collected before World War I. An overview of the vegetation of the western shore in the 1930s was presented by Eichwald (1939); the vegetation of the eastern shore in the same period was discussed by Pastak (1936). Although the last work focuses on the species occurring on the shore and in shallow water, it represents a valuable addition to other lists, which have paid less attention to the species of the flooded shore zone. In 1951, the vegetation of L. Võrtsjärv was studied by $\mathrm{H}$. Tuvikene, whose summary data have not so far been published. A good description of the distribution areas of the vegetation can be found in a paper on the distribution of zoobenthos in L. Vorrtsjärv, which was completed by Ristkok \& Ruse (1962) on the basis of the data from 1959-60. In 1965-67, the littoral of L. Võrtsjärv was studied by A. Mäemets (1973); the results of this research served as a basis of the chapter "Higher vegetation" in the monograph on Lake Võrtsjärv. A. Mäemets conducted repeat observations in 1985-86, but she was unable to study the whole shoreline again. The unpublished data of 1985-86 cover the lake's southern part from the mouth of the Ôhne River to Konnaküla (Fig. 3). In 1995 and 1997, the vegetation of the whole lake was thoroughly investigated by T. Feldmann, who dealt mainly with factors determining the distribution of the vegetation. Feldmann (2000) and P. Nõges (Nõges et al., 1999; Nõges \& Feldmann, 1999) compared the most recent data with the floristic list of A. Mäemets (1973), and P. Nõges (Nõges et al., 2001) juxtaposed new data with the floristic list of von zur Mühlen.

The data of different authors are summarized in Table 1. In some cases older determinations are preceded by later, more reliable identifications. The abundance estimates are given for species supplied with comments in the text; the other species are denoted with "o" (i.e. definite occurrence). The following notes should be made about the data of the different authors:

1. The survey by Mühlen (Mühlen \& Schneider, 1920) was completed in parallel with other hydrobiological studies on L. Võrtsjärv, which were carried out from a boat. Therefore Mühlen admitted that the species growing at the water's edge were less represented and some infrequent species among reeds might have remained unnoticed.

2. Pastak (1936) made her observations walking along the shore and therefore data for submergent species and for species occurring among reeds are almost lacking.

3. Eichwald's (1939) observations carried out on the western shore reflect more adequately its southern part. Potamogeton gramineus, highly characteristic of the northern part, is absent from his list. 
4. The unpublished list of $\mathrm{H}$. Tuvikene does not present abundance estimates, because relevant data have evidently got lost. The list lacks several species, which suggests that only a few regions were visited.

5. The abundance estimates included in A. Mäemets's data from 1965-67 are presented as intermediate between the estimates given in the table comparing L. Peipsi and L. Võrtsjärv, compiled by her, and those given in the monograph on L. Võrtsjärv. Note that the estimates for 1985-86 apply only to the studied lake part.

6. The work of Feldmann (2000) presents the data on frequency, i.e. the ratio of the number of shore stretches having the species to the number of investigated shore stretches, and relative abundance, i.e. the ratio of the number of shoots of the species in the experimental plots to the number of shoots for all species in the experimental plots. In many cases these indices are different, and the estimates in Feldmann's species list represent a compromise between these two estimates. Use has been made of the remarks about changes that have taken place in species distribution, as presented in his MSc thesis. Twenty-six species of his floristic list, which I do not regard as specifically characteristic of the shore of L. Vortsjärv, such as Knautia arvensis, Plantago major, etc., have been excluded from Table 1.

7. The author's observations in the 1996-2001 excluded the NW coast of the lake, so the estimates are less representative in comparison with those of Feldmann (2000).

The group of emergent plants, especially hygrophytes, belonging to three major families (Poaceae, Cyperaceae, and Juncaceae), are least studied. Identification of these species is troublesome, and when making observations from a boat, they can be neglected by hydrobotanists. In this respect, the species list for L. Võrtsjärv (as well as for L. Peipsi) is rather incomplete. An attempt was made to fit verbal abundance estimates in a unified system with four classes: $\mathrm{x}$ - rare; $\mathrm{xx}$ - occasional, in some places; $\mathrm{xxx}$ - frequent; $\mathrm{xxxx}$ - very frequent. However, this procedure appears quite subjective for differently presented data. The estimates for a particular lake part have not been extended to overall (proportional) estimates.

\section{RESULTS AND DISCUSSION}

\section{Main vegetation regions and their changes}

A total of 40 taxa of hydrophytes and amphibious plants have been identified in L. Võrtsjärv (Table 1). In comparison with 50 taxa of L. Peipsi, this is quite a large number considering the smaller size and turbid water of the former. Also 100 years ago the transparency of L. Võrtsjärv rarely exceeded $1 \mathrm{~m} \mathrm{SD}$ in summer (Mühlen \& Schneider, 1920). The number of taxa included in the group of emergent plants depends on the purpose of observation and on the methods applied by investigators, especially regarding the species of the flooded shore zone. In L. Võrtsjärv the number of emergent species is over 70; however, 
Table 1. List of macrophyte taxa of Lake Võrtsjärv with their occurrence in different periods

\begin{tabular}{|c|c|c|c|c|c|c|c|c|c|}
\hline Taxon & 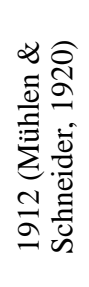 & 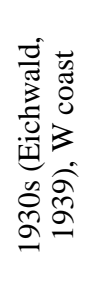 & 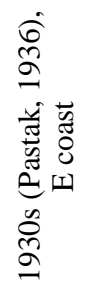 & 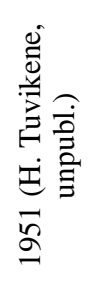 & 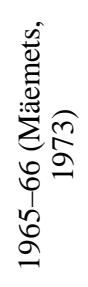 & 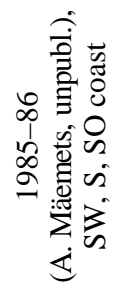 & 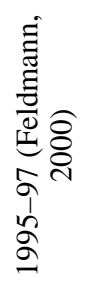 & 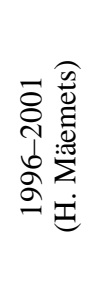 & $\begin{array}{l}\text { Growth areas in } \\
\text { L. Võrtsjärv }\end{array}$ \\
\hline \multicolumn{10}{|l|}{ SUBMERGED PLANTS } \\
\hline Chara contraria A. Br. (foetida by Eichwald) & & $\mathrm{xx}$ & & & $\mathrm{xxx}$ & & & & evw fr \\
\hline Chara spp. & $\mathrm{xx}$ & & & o & & & & & earl fr in $S p$ \\
\hline Nitellopsis obtusa (Desv. in Lois.) Gr. & & & & & $\mathrm{xx}$ & & & & mass in S p 1960s \\
\hline $\begin{array}{l}\text { Drepanocladus aduncus (Hedw.) Moenkem. f. } \\
\text { capillifolia }\end{array}$ & & & & & $\mathrm{x}$ & & & & at Vaibla rl \\
\hline Fontinalis antipyretica DC. & & & & & & $\mathrm{x}$ & & & at Pähksaar I \\
\hline R. circinatus Sibth. & & $\mathrm{xx}$ & & & $\mathrm{xx}$ & $\mathrm{xx}$ & $\mathrm{x}$ & $\mathrm{x}$ & mainly in $\mathrm{S} \mathrm{p}$ \\
\hline R. trichophyllus Chaix (s.l.) & o & & & & & & & $\mathrm{x}$ & at Jõesuu 2001 \\
\hline Ceratophyllum demersum $\mathrm{L}$. & & & & & $\mathrm{x}$ & $\mathrm{xxx}$ & $\mathrm{xxx}$ & $\mathrm{xx}$ & incr, mass in $\mathrm{S} \mathrm{p}$ \\
\hline Myriophyllum spicatum L. & & & & o & $\mathrm{xx}$ & $\mathrm{xxx}$ & $\mathrm{xxxx}$ & $\mathrm{xxxx}$ & dom evw \\
\hline M. verticillatum $\mathrm{L}$. & & & & & & $\mathrm{xx}$ & $\mathrm{xx}$ & $\mathrm{xx}$ & mainly in $\mathrm{S} \mathrm{p}$ \\
\hline Myriophyllum sp. & $\mathrm{xx}$ & & & & & & & & \\
\hline Callitriche hermaphroditica Jusl. & & & & & $\mathrm{x}$ & & & & at Pähksaar I \\
\hline Hottonia palustris $\mathrm{L}$. & & & & & $\mathrm{x}$ & & & $\mathrm{x}$ & in $\mathrm{S} \mathrm{p} \mathrm{\&} \mathrm{rl}$ \\
\hline Utricularia vulgaris $\mathrm{L}$. & & & & & $\mathrm{x}$ & & $\mathrm{x}$ & $\mathrm{xx}$ & sc \\
\hline Elodea canadensis $\mathrm{L}$. & $\mathrm{xx}$ & $\mathrm{xxx}$ & & & $\mathrm{xxx}$ & $\mathrm{x}$ & & $\mathrm{x}$ & earl $\mathrm{S} p, \mathrm{~W}$ sh, now sc \\
\hline$\underline{\text { Stratiotes aloides } \mathrm{L} .}$ & & $\mathrm{xx}$ & & & $\mathrm{xx}$ & $\mathrm{xx}$ & & $\mathrm{x}$ & $\mathrm{Sp}$ \\
\hline Potamogeton alpinus Balb. & & $\mathrm{xx}$ & & & & & & & $\mathrm{Sp}$ \\
\hline P. crispus $\mathrm{L}$. & & $\mathrm{xx}$ & & & $\mathrm{x}$ & $\mathrm{x}$ & & & $\mathrm{S}, \mathrm{W} \mathrm{p}$, in places \\
\hline P. filiformis Pers. & & & & & $\mathrm{x}$ & $\mathrm{x}$ & & $\mathrm{xx}$ & at N, E sh \\
\hline P. friesii Rupr. & & & & & $\mathrm{x}$ & & & & \\
\hline P. gramineus $\mathrm{L}$. & $\mathrm{xxx}$ & ? & $\mathrm{xx}$ & $\mathrm{o}$ & $\mathrm{xxx}$ & $\mathrm{xx}$ & $\mathrm{xxx}$ & $\mathrm{xxx}$ & fr at $N$ and $E$ sh \\
\hline P. lucens L. & $\mathrm{xx}$ & $\mathrm{xxx}$ & & $\mathrm{o}$ & $\mathrm{xxx}$ & $\mathrm{xxx}$ & $\mathrm{xxx}$ & $\mathrm{xxx}$ & mass in SE, $\mathrm{S}, \mathrm{W}$ p, fr \\
\hline
\end{tabular}


Table 1 continued

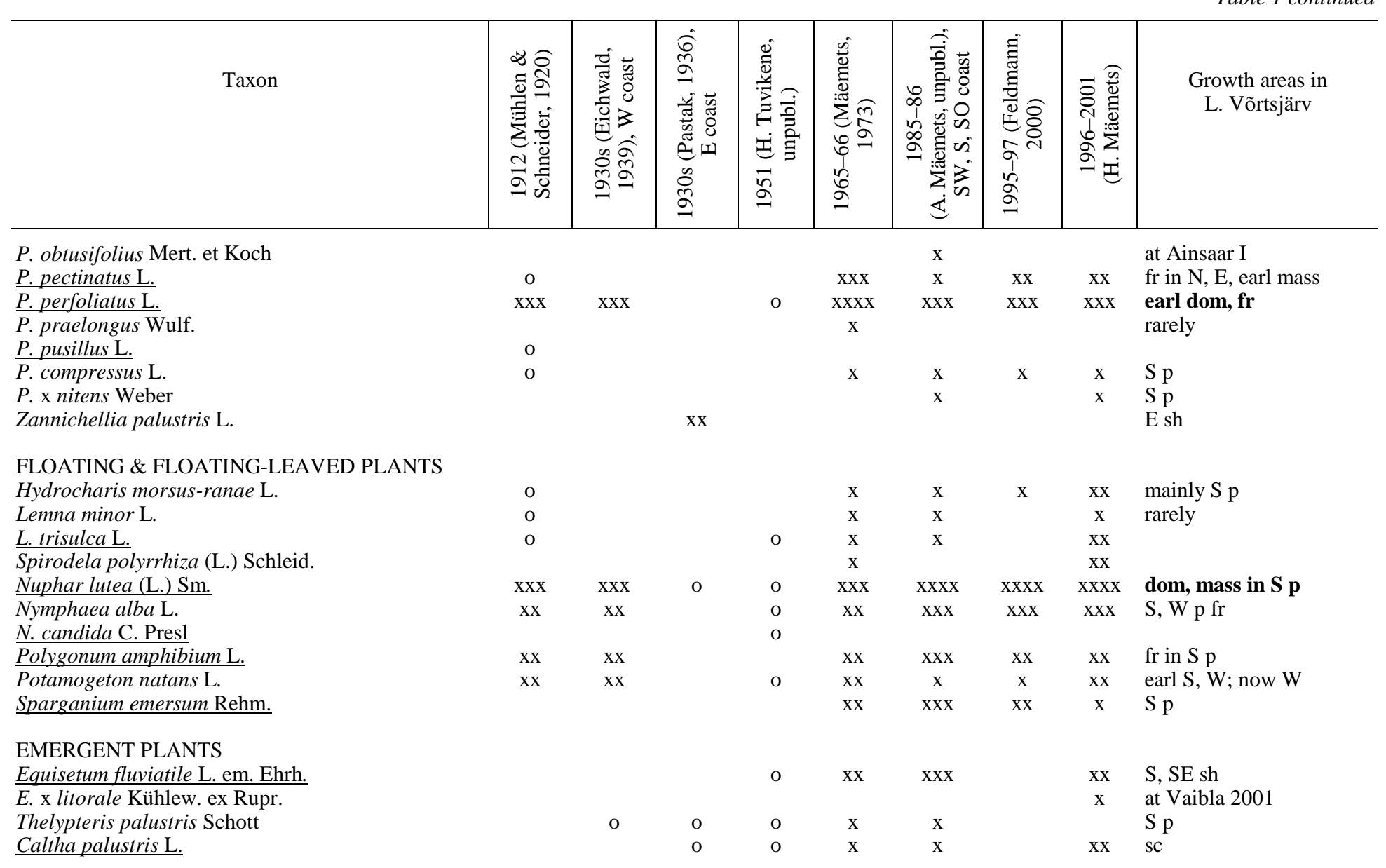




\begin{tabular}{|c|c|c|c|c|c|c|c|c|c|}
\hline Taxon & 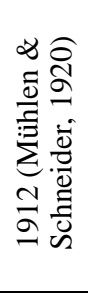 & 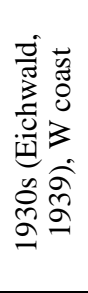 & 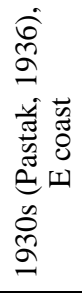 & 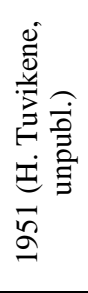 & 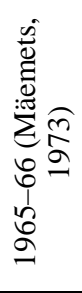 & 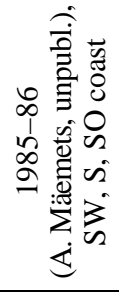 & 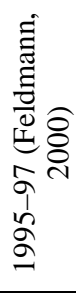 & 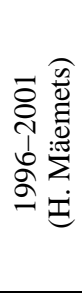 & $\begin{array}{l}\text { Growth areas in } \\
\text { L. Võrtsjärv }\end{array}$ \\
\hline Ranunculus lingua $\mathrm{L}$. & $\mathrm{xx}$ & $\mathrm{xx}$ & $\mathrm{o}$ & $\mathrm{o}$ & $\mathrm{x}$ & $\mathrm{xx}$ & & & $\mathrm{sc}$ \\
\hline R. reptans $\mathrm{L}$. & $\mathrm{o}$ & & $\mathrm{xxx}$ & & $\mathrm{xxx}$ & & $\mathrm{x}$ & $\mathrm{xx}$ & fl sh z, N, E p \\
\hline R. sceleratus L. & & & & & & & & $\mathrm{x}$ & \\
\hline Comarum palustre L. & o & $\mathrm{o}$ & & & $\mathrm{x}$ & $\mathrm{x}$ & & & \\
\hline Potentilla anserina $\mathrm{L}$. & & & $\mathrm{xx}$ & & $\mathrm{xx}$ & & $\mathrm{xx}$ & $\mathrm{xx}$ & fl sh z, N, E p \\
\hline Lathyrus palustris L. & & & & & & & & $\mathrm{x}$ & at Pähksaar I \\
\hline Lythrum salicaria $\mathrm{L}$. & & & $\mathrm{xx}$ & o & $\mathrm{xx}$ & $\mathrm{xx}$ & $\mathrm{xx}$ & $\mathrm{xxx}$ & fl sh z, sc \\
\hline Cicuta virosa $\mathrm{L}$. & o & & $\mathrm{o}$ & & $\mathrm{x}$ & $\mathrm{x}$ & & $\mathrm{xx}$ & mainly $\mathrm{S} \mathrm{p}$ \\
\hline Oenanthe aquatica (L.) Poir. & $\mathrm{o}$ & & o & & $\mathrm{xx}$ & $\mathrm{xxx}$ & $\mathrm{xx}$ & $\mathrm{xx}$ & fr in $S \mathrm{p}$, elsew sc \\
\hline Sium latifolium L. & $\mathrm{xx}$ & $\mathrm{xx}$ & $\mathrm{xx}$ & o & $\mathrm{xx}$ & $\mathrm{xxx}$ & $\mathrm{xx}$ & $\mathrm{xx}$ & fr in $S \mathrm{p}$, elsew sc \\
\hline Galium palustre L. & & & $\mathrm{xx}$ & & $\mathrm{xx}$ & & $\mathrm{x}$ & $\mathrm{xx}$ & fl sh z \\
\hline Menyanthes trifoliata $\mathrm{L}$. & o & & & & $\mathrm{xx}$ & & & & mainly S p \\
\hline Myosotis scorpioides L. & o & $\mathrm{o}$ & & & $\mathrm{x}$ & & $\mathrm{x}$ & $\mathrm{xx}$ & fl sh z \\
\hline Symphytum officinale L. & & & & & & & $\mathrm{x}$ & $\mathrm{xx}$ & \\
\hline Solanum dulcamara L. & & & $\mathrm{xx}$ & & & $\mathrm{x}$ & & $\mathrm{xx}$ & \\
\hline Scutellaria galericulata $\mathrm{L}$. & & & $\mathrm{o}$ & & & & $\mathrm{x}$ & $\mathrm{xx}$ & \\
\hline Lycopus europaeus L. & & & $\mathrm{o}$ & & $\mathrm{xx}$ & $\mathrm{x}$ & & $\mathrm{xx}$ & fl sh z \\
\hline Mentha aquatica $\mathrm{L}$. & & & & & $\mathrm{x}$ & & $\mathrm{x}$ & $\mathrm{xx}$ & \\
\hline Stachys palustris L. & & & & & & $\mathrm{x}$ & $\mathrm{x}$ & $\mathrm{xxx}$ & \\
\hline Hippuris vulgaris $\mathrm{L}$. & & & & & $\mathrm{x}$ & & & $\mathrm{xx}$ & \\
\hline Rorippa amphibia (L.) Bess. & o & & $\mathrm{o}$ & & $\mathrm{xx}$ & $\mathrm{xxx}$ & $\mathrm{xxx}$ & $\mathrm{xxx}$ & fr in $S p$, shel places \\
\hline Achillea ptarmica $\mathrm{L}$. & & & & & & & $\mathrm{x}$ & $\mathrm{xx}$ & \\
\hline A. salicifolia Besser & & & & & & & $\mathrm{x}$ & $\mathrm{xx}$ & \\
\hline Bidens cernua L. & & & & & & & & $\mathrm{x}$ & \\
\hline B. tripartitus L. & & & & & & & & $\mathrm{xx}$ & \\
\hline Cirsium arvense (L.) Scop. & & & & & & & $\mathrm{x}$ & $\mathrm{xx}$ & \\
\hline
\end{tabular}


Table 1 continued

\begin{tabular}{|c|c|c|c|c|c|c|c|c|c|}
\hline Taxon & 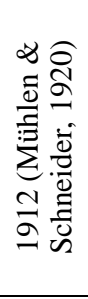 & 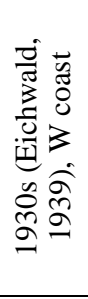 & 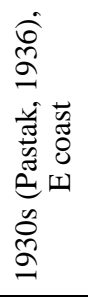 & 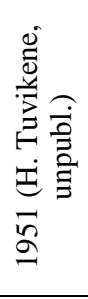 & 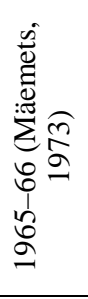 & 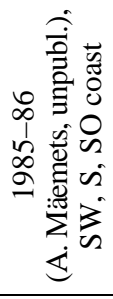 & 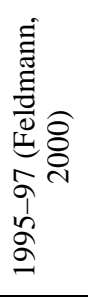 & 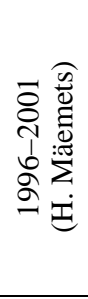 & $\begin{array}{l}\text { Growth areas in } \\
\text { L. Võrtsjärv }\end{array}$ \\
\hline Tussilago farfara $\mathrm{L}$. & & & & & & & $\mathrm{x}$ & $\mathrm{xx}$ & \\
\hline Senecio paludosus $\mathrm{L}$. & & & & & & & & $\mathrm{xx}$ & \\
\hline Myosoton aquaticum (L.) Moench & & & & & & & & $\mathrm{xx}$ & \\
\hline Rumex hydrolapathum Huds. & & & & & $\mathrm{x}$ & $\mathrm{x}$ & & & more in S p \\
\hline Rumex maritimus L. & & & & & & & $\mathrm{x}$ & $\mathrm{xx}$ & E sh \\
\hline Lysimachia nummularia $\mathrm{L}$. & & & & & $\mathrm{x}$ & & & $\mathrm{xx}$ & \\
\hline L. thyrsiflora $\mathrm{L}$. & & o & & & $\mathrm{x}$ & $\mathrm{xx}$ & & $\mathrm{x}$ & $\mathrm{Sp}, \mathrm{sc}$ \\
\hline$\overline{\text { L. vulgaris } \mathrm{L} \text {. }}$ & & & & $\mathrm{o}$ & & $\mathrm{x}$ & $\mathrm{x}$ & $\mathrm{xxx}$ & \\
\hline A. plantago-aquatica $\mathrm{L}$. & $\mathrm{xx}$ & & & & $\mathrm{x}$ & $\mathrm{xxx}$ & $\mathrm{xx}$ & $\mathrm{xxx}$ & sc, mass in Maapera Bay \\
\hline Sagittaria sagittifolia $\mathrm{L}$. & $\mathrm{xx}$ & & & $\mathrm{o}$ & $\mathrm{xxx}$ & $\mathrm{xxx}$ & $\mathrm{xxx}$ & $\mathrm{xxx}$ & in est, mass in $\mathrm{S} \mathrm{p}$ \\
\hline$\overline{\text { Iris pseudacorus } \mathrm{L} \text {. }}$ & $\mathrm{xx}$ & $\mathrm{xx}$ & & & $\mathrm{x}$ & $\mathrm{x}$ & & $\mathrm{xx}$ & decr, sc \\
\hline Butomus umbellatus $\mathrm{L}$. & $\mathrm{xx}$ & & $\mathrm{o}$ & & $\mathrm{xxx}$ & $\mathrm{xxx}$ & $\mathrm{xxx}$ & $\mathrm{xxx}$ & at infl, $\mathrm{S}$ and $\mathrm{W}$ sh \\
\hline Juncus ambiguus (coll.) Guss. & & & & & & & & $\mathrm{x}$ & \\
\hline J. articulatus L. (J. alpinus by Mühlen) & $\mathrm{xxx}$ & & & & $\mathrm{xxx}$ & & & $\mathrm{xx}$ & fl sh z \\
\hline J. compressus Jacq. & & & $\mathrm{o}$ & & & & & & fl sh z \\
\hline J. nodulosus Wahlenb. & & & $\mathrm{xxx}$ & & $\mathrm{xx}$ & & $\mathrm{x}$ & $\mathrm{xx}$ & fl sh z \\
\hline Eleocharis acicularis Roem. et Schult. & $\mathrm{xx}$ & & $\mathrm{xxx}$ & & $\mathrm{xxx}$ & $\mathrm{x}$ & & $\mathrm{xx}$ & $\mathrm{fl} \mathrm{sh} \mathrm{z}$, more in $\mathrm{E} p$ \\
\hline E. palustris (L.) Brown em. Roem. et Schult. & $\mathrm{xx}$ & & o & o & $\mathrm{xx}$ & $\mathrm{xx}$ & $\mathrm{x}$ & $\mathrm{xxx}$ & in $\mathrm{N}$ and $\mathrm{E} \mathrm{p} \mathrm{fr}$ \\
\hline $\begin{array}{l}\text { E. uniglumis (Link.) Schult. } \\
\text { Eriophorum angustifolium Honck. (latifolium by } \\
\text { Mühlen) }\end{array}$ & $\mathrm{xxx}$ & & $\mathrm{xx}$ & & $\mathrm{xxx}$ & $\mathrm{xx}$ & & $\mathrm{xx}$ & $\begin{array}{l}\mathrm{N} \text { and } \mathrm{E} \text { sh } \\
\mathrm{N} \text { and } \mathrm{E} \text { sh }\end{array}$ \\
\hline $\begin{array}{l}\text { Schoenoplectus lacustris (L.) Palla } \\
\text { S. sylvaticus L. }\end{array}$ & $\mathrm{xxx}$ & $\mathrm{xxx}$ & o & & $\mathrm{xxx}$ & $\mathrm{xxxx}$ & $\mathrm{xxx}$ & $\begin{array}{c}\mathrm{xxx} \\
\mathrm{xx}\end{array}$ & ab in $S p$, elsew sc \\
\hline Carex acuta $\mathrm{L}$. & & & o & & & & $\mathrm{x}$ & $\mathrm{xxx}$ & \\
\hline $\begin{array}{l}\text { C. acutiformis Ehrh. } \\
\text { C. elata Bell. }\end{array}$ & o & & $\mathrm{xxx}$ & & & & & $\mathrm{xx}$ & \\
\hline
\end{tabular}




\begin{tabular}{|c|c|c|c|c|c|c|c|c|c|}
\hline Taxon & 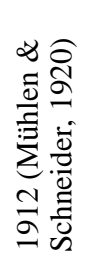 & 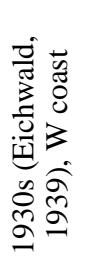 & 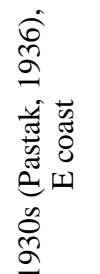 & 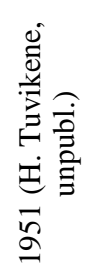 & 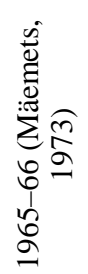 & 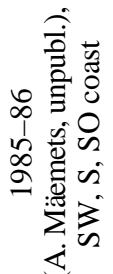 & 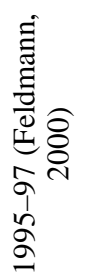 & 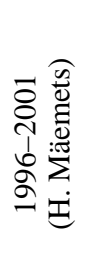 & $\begin{array}{l}\text { Growth areas in } \\
\text { L. Võrtsjärv }\end{array}$ \\
\hline
\end{tabular}

C. hirta $\mathrm{L}$.

C. lasiocarpa Ehrh.

C. nigra (L.) Reichard

C. pseudocyperus $\mathrm{L}$.

C. rostrata Stok.

C. vesicaria $\mathrm{L}$.

C. viridula Michx.

Agrostis stolonifera $\mathrm{L}$.

Calamagrostis canescens (Weber) Roth

C. neglecta subsp. neglecta (Ehrh.) P. Gaertn.

Glyceria maxima (Hartm.) Holmb.

G. fluitans (L.) R. Brown

Phalaris arundinacea L

Phragmites australis (Cav.) Trin ex Steud.

Scolochloa festucacea (Willd.) Link.

Acorus calamus L.

Sparganium erectum L. (coll.)

Typha angustifolia $\mathrm{L}$.

T. latifolia $\mathrm{L}$. o

$\mathrm{x}$

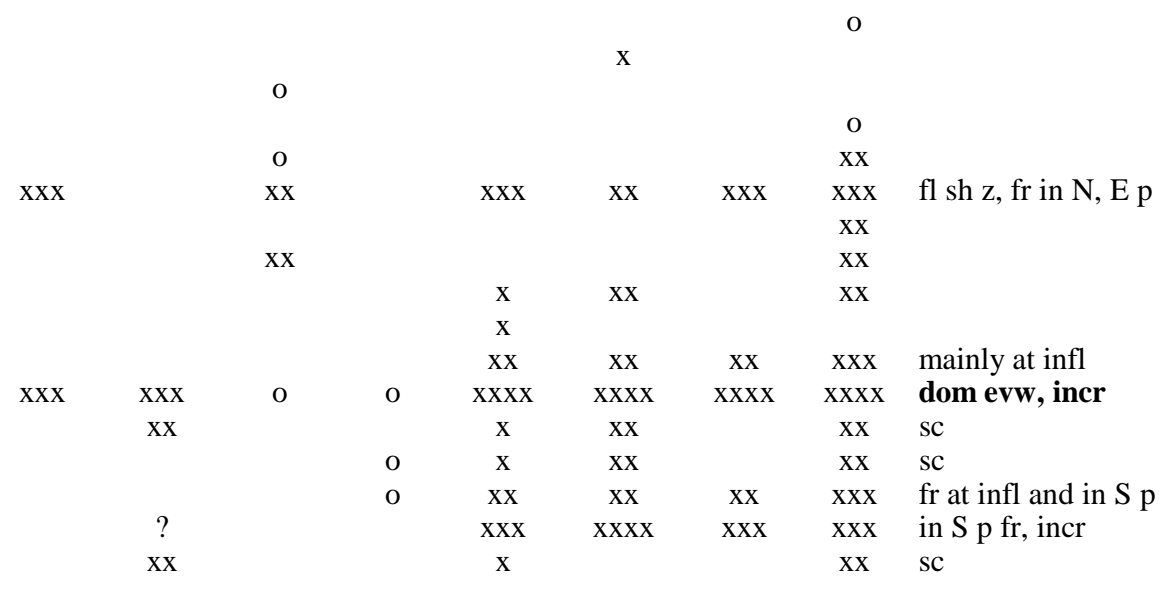

Abbreviations: N, S, NW etc., directions of the compass; ab, abundant(ly); decr, decreasing; dom, dominating; earl, earlier; elsew, elsewhere; est, estuary; evw, everywhere; fl, flooded; fr, frequent(ly); I, island; incr, increasing; infl, inflow(s); mass, in masses; p, part; rl, rivulet(s); sc, scattered; shel, sheltered; sh, shore; $\mathrm{z}$, zone.

Frequency (4 points): $\mathrm{x}$ - rare; $\mathrm{xx}$ - occasional; $\mathrm{xxx}$ - frequent; $\mathrm{xxxx}$ - very frequent.

Underlined: common species of L. Peipsi and other large lakes of the NW part of the Russian Platform (Raspopov, 1985). 
inclusion of the plants of the higher shore belt would extend their list. Extensive denuded areas in the littoral are characteristic of large shallow lakes and their flora is rich in pioneer species incapable of competing in climax associations.

Like the southern part of L. Peipsi, the southern part of L. Võrtsjärv has always been more eutrophic and richer in vegetation than the rest of the lake. In both lakes, the highest pollution load originates from the discharge into the southern tip. In L. Võrtsjärv, exuberant growth of vegetation is favoured also by a significantly weaker effect of the wind and waves, as well as by the more restricted spread of sedimented organic material, resulting from the small width of the lake in its southern part. The wider northern part of the lake, particularly areas affected by dominating westerly and southwesterly winds, have always been poorer in vegetation, as was demonstrated also by Feldmann (2000). Furthermore, pollution and organic sediments from inflows are more dispersed in the wider lake part. Despite the inhibiting effect of the wind and waves, reeds have occupied ever more extensive shore stretches around both large lakes during the last 40 years. While only one of the 17 von zur Mühlen's photos from the early 20th century displays reeds, and the presence of moving dunes is still evident, then on the map by Feldmann (2000) from the end of that century (Fig. 6) reeds occupy already most of the shoreline, leaving only a few shorter or longer stretches free. According to Ristkok \& Ruse (1962) the inlet of Vehendi (for location see Fig. 3) was devoid of reeds at the end of the 1950s. However, A. Mäemets found reeds there in the mid-1960s (Fig. 6). The spread of reeds is the most significant consequence of eutrophication, coinciding with a low-water period in 1965-76 (Fig. 5) and occurring both in L. Peipsi and L. Võrtsjärv.

Regardless of the changes, the vegetation reveals differences between the southern and northern parts of L. Võrtsjärv; the demarcation line runs along the inlet of Tarvastu-Tondisaar Island-Rannaküla (Fig. 3). Besides reeds, characteristic plants in the northern part include those tolerating water level changes, such as Potamogeton gramineus, Agrostis stolonifera, Eleocharis uniglumis, Juncus articulatus, and J. nodulosus. The abundance of amphibious species was particularly high earlier, when the littoral zone was open. According to Feldmann (2000), P. gramineus occupies now the 23rd place in frequency and the 6th place in relative abundance (see Methods). The southern part is characterized by a regular occurrence of Schoenoplectus lacustris as well as by the presence of dense, partly impassable reeds and large stands of Nuphar lutea. The species composition in the southern part is typical of eutrophic-hypertrophic water bodies and has undergone more profound changes compared with the species composition of the northern regions. The most significant feature is the disappearance of the formerly quite widespread Chara contraria and Nitellopsis obtusa, which took place probably in the 1970s. Elodea canadensis, a nuisance for fishermen in the early 20th century, has almost disappeared, whereas Ceratophyllum demersum occurs abundantly. The formerly quite abundant Stratiotes aloides has almost disappeared as well. The most prominent change in the vegetation of the whole lake is the turning of Myriophyllum spicatum into a submergent dominant (Fig. 6). According to Feldmann (2000), this species comes 
first in frequency but, due to its sparse stands, fifth in relative abundance. Potamogeton lucens and P. perfoliatus, predominating in deeper water until recently, are surpassed by M. spicatum with respect to both parameters. The appearance of Typha angustifolia and its increase in the second half of the 20th century are noteworthy.

\section{Specific features of the macroflora of $L$. Võrtsjärv}

The macroflora of L. Võrtsjärv has developed during several millennia in close association with the macroflora of L. Peipsi. In some periods, definitely in the late glacial but possibly also later, the lakes of the Vooremaa drumlin area formed part of the Peipsi-Võrtsjärv lake system (Fig. 7). An evidence of this might be the occurrence of Potamogeton filiformis in lakes Peipsi and Võrtsjärv as well as in the larger lakes of the Vooremaa district, e.g. Saadjärv and Kuremaa. Both earlier and contemporary researchers regard this alkaliphilous species as a relict from the late glacial (Samuelsson, 1934; Preston \& Croft, 1997). Chara contraria and Nitellopsis obtusa have grown or are growing in L. Peipsi and L. Võrtsjärv as well as in most lakes of Vooremaa. The list of the macroflora of L. Võrtsjärv does not contain species that have not been found or are not found in the littoral or in the estuaries of the inflows of L. Peipsi. The differences in the macroflora between these lakes involve rare species, whose number is larger in L. Peipsi. The species not found in L. Võrtsjärv are Alisma gramineum C. Ch. Gmel., Elatine hydropiper L., Cyperus fuscus L., Scirpus radicans Schkuhr., Potamogeton rutilus Wolfg., $P$. trichoides Cham. et Schlecht., and already extinct in L. Peipsi Isoëtes echinospora Durieu and Subularia aquatica L. The absence of Potamogeton berchtoldii Fieber, rather common in Estonian lakes, is doubtful. Evidently, this species has remained undetected. The vegetation of the shallowwater and the temporarily flooded shore zones of L. Peipsi, which includes the above-mentioned species, is floristically the richest. The shores of L. Võrtsjärv lack Petasites spurius (Retz.) Reichenb. and Leymus arenarius (L.) Hochst., characteristic of the dunes of L. Peipsi (Kalda, 2000). Since moraines on the shores of L. Võrtsjärv are particularly rich in lime (Kongo, 1973), there occur species typical of limestone areas, e.g. Rubus caesius L. The alkaliphilous vegetation of the water's edge and submerged species contrast with Rumex acetosa $\mathrm{L}$. meadows lying close to the shore in the southern dune areas. The submerged vegetation reveals some differences in occurrence of some rarer species in L. Peipsi, but most differences involve the proportion of common species. Potamogeton perfoliatus has always been a dominant in L. Peipsi, while the frequency of $P$. lucens has been higher periodically. In L. Võrtsjärv, $P$. lucens has always been frequent, while the former dominant $P$. perfoliatus has been surpassed by Myriophyllum spicatum in recent decades. P. gramineus has occupied an important place in L. Peipsi and L. Võrtsjärv during all known history, while P. pectinatus seems to have declined somewhat in L. Võrtsjärv. Although quantitative data originate from different periods and are therefore not 


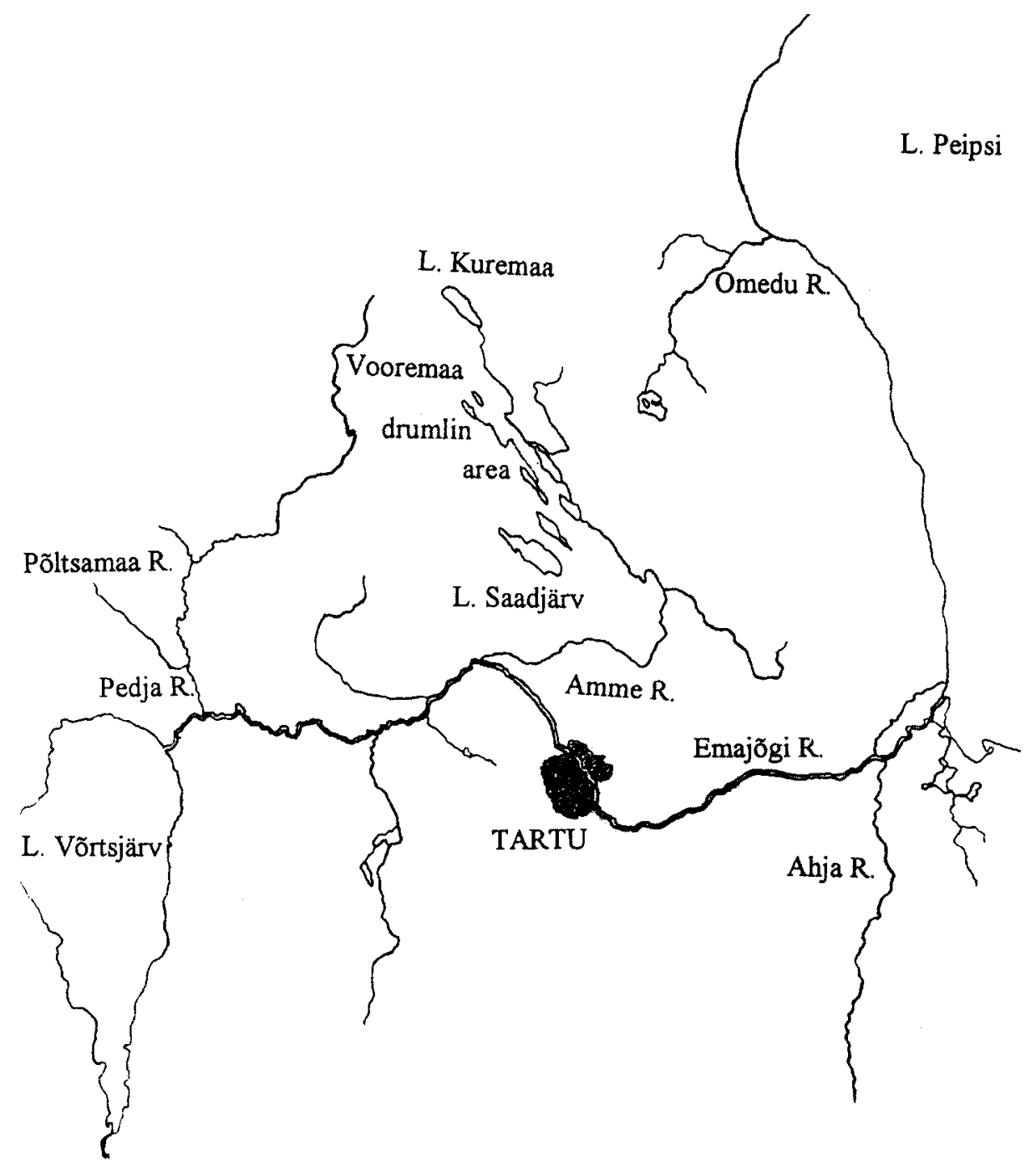

Fig. 7. A part of the former Peipsi-Võrtsjärv lake system area.

quite comparable, the reeds of L. Võrtsjärv are characterized by lower density and biomass compared with those of L. Peipsi or Matsalu Bay of the Baltic Sea in West Estonia (Ksenofontova, 1989; Sudnitsõna, 1990; Feldmann, 2000). One reason for this difference may be the location of the experimental plots, which excludes exuberant reeds growing beyond the water's edge (Feldmann, 2000). However, the sparsity of reeds (in water) has been pointed out by earlier botanists who have studied the lake. When at the time of the studies of von zur Mühlen and even as late as the first studies of A. Mäemets the reed belt distant from the shore was a specific feature of L. Võrtsjärv, then at present it is growing ever closer to 
the shore. In the environs of the Limnological Station (Fig. 3) the open area between the water's edge and the reed belt has decreased at least by half or has disappeared in places.

Lakes Võrtsjärv and Peipsi share several species of the higher belt of the shore zone, which are not common on the territory of Estonia. These are Inula britannica, Symphytum officinale, and Achillea salicifolia. Noteworthy is the occurrence of Juncus nodulosus in the flooded zone of L. Võrtsjärv. The main distribution area of this species is the West Estonian seashore. Lakes Võrtsjärv, Peipsi, Ladoga, and Onega as well the other five large lakes (Ilmen, Beloe, Kubenskoe, Vozhe, Lacha) of northwestern Russia share 19 commonest macrophyte species and, in addition, 12 species that have not been found in one or two lakes (both underlined in Table 1) (Raspopov, 1985; Mäemets \& Mäemets, 2000). Of these 12 species, Potamogeton pusillus and Nymphaea candida have not been recorded in L. Võrtsjärv presently. Overgrowing of shallow water with reeds decreases the species of the flooded zone, characteristic of large lakes. Eleocharis acicularis, opulent in the northern part according to Mühlen (1920), is absent from the list of Feldmann, based on the material of transects. This species occurs in places, but it may have remained unnoticed due to its decreased abundance.

\section{Reasons for changes in the vegetation of $L$. Võrtsjärv in the course of eutrophication and comparison with $L$. Peipsi}

The main reason for the extinction of Charophyta in L. Võrtsjärv is evidently the increase in water phosphorus content. Summarizing the estimations of several authors as well as his personal data, Sviridenko (2000) points out the importance of a low level of this nutrient. According to Sviridenko, Charophyta are relatively tolerant of low water transparency, as confirmed by the example of L. Võrtsjärv. The disappearance of Chara contraria and Nitellopsis coincides with the period of the highest nutrient loading in the 1970s. The decline of Elodea canadensis and Stratiotes aloides is more difficult to explain. The former species is able to accumulate large quantities of phosphorus from sediment, which was revealed by our observations. The extensive distribution of Elodea after its invasion in the first half of the 20th century was later replaced by a decline in many lakes of Estonia. The decrease in Elodea in L. Võrtsjärv can be partly explained by this general trend. However, both these species may suffer for anoxic conditions in sediment. Feldmann (2000) refers to authors who have indicated the unavailability of iron to Stratiotes, and the toxicity of sulphides and ammonium formed on reduction (Smolders et al., 1996). Prevalence of unrooted Ceratophyllum is characteristic in such conditions, as can be seen in the southern part of L. Vortsjärv.

Finding out the reasons for the appearance of Myriophyllum verticillatum simultaneously with the expansion of M. spicatum in the 1980s requires further investigation on the ecology of M. verticillatum. This species seems to be 
calcicolous in our lakes as well as in lakes of other regions (Preston \& Croft, 1997), but it may occur in relatively different water bodies, including shallow muddy creeks. The recent predominance of $M$. spicatum in L. Võrtsjärv may be a consequence of phosphorus enrichment in anoxic sediment layers. The functioning of the lake as a biological purification reservoir of wastewater, discharged from surrounding settlements and fertilized agricultural areas over decades, has resulted in phosphorus accumulation (Haberman et al., 1998; Nõges et al., 1998). M. spicatum withstands the action of waves (Schmieder, 1998) and displays switchover from the use of nutrients from sediment to water, depending on their concentrations (Mantai \& Newton, 1982). The disappearance of this species from L. Constance in the period of the highest nutrient loading (data from 1978) and its reappearance in the 1990s after an essential decrease in phosphorus inflow are significant (Schmieder, 1998). A. Mäemets (1973) pointed out the fast colonization by $M$. spicatum as a consequence of low-water periods and the abundance of this species after the exceedingly low water level in 1996 confirms this opinion. The decline of eutrophication-tolerant Potamogeton pectinatus in L. Võrtsjärv may have resulted from the presence of restricted reed-free shallow-water areas in its former habitats.

Reeds have expanded in the course of eutrophication in both L. Võrtsjärv and L. Peipsi. Owing to the differences in the size, turnover time, and pollution of the two lakes, the expansion of reeds in L. Vorrtsjärv was the fastest in the 1960s and in L. Peipsi in the 1970s. A maximum of species richness was reached almost in the same period, in 1965-70. The species inhabiting the open (reed-free) flooded zone have been decreasing similarly in recent decades. The frequency of the dominating submerged species of L. Peipsi, Potamogeton perfoliatus, has not changed during eutrophication, and the other common species of L. Peipsi, $P$. pectinatus and P. gramineus, occur frequently today as well. Changes in the macroflora of L. Võrtsjärv as a possible pattern for L. Peipsi, for example replacement of $P$. perfoliatus by Myriophyllum spicatum, cannot be excluded. However, basic changes in both lakes have taken place more or less simultaneously and differences in the abundance of common species may persist in the future.

\section{ACKNOWLEDGEMENTS}

This survey was completed in memory of Aime Mäemets, who spent 35 years of her life here, on the coast of Lake Võrtsjärv. The paper was inspired by Juta Haberman, who deserves my sincere gratitude. I am grateful to Heljo Tuvikene, Tõnu Feldmann and Peeter Nõges, who permitted me to use their data on the macrovegetation of L. Võrtsjärv, as well as to Tiiu Kull and Thea Kull for the provision of data on the distribution areas of several species. The English manuscript was kindly revised by Ester Jaigma. The research was partly supported by Contract No. EVK1-CT-2000-000076 Mantra-East. 


\section{REFERENCES}

Coops, H. \& Doef, R. W. 1996. Submerged vegetation development in two shallow, eutrophic lakes. Hydrobiologia, 340, 115-120.

Eichwald, K. 1939. Võrtsjärve taimestik. In Viljandimaa I., pp. 57-59. EKS Kirjastus, Tartu.

Feldmann, T. 2000. Kõrgema veetaimestiku levik Võrtsjärves ja seda määravad tegurid. MSc thesis, University of Tartu.

Haberman, J., Nõges, P., Nõges, T., Pihu, E., Kangur, K., Kangur, A. \& Kisand, V. 1998. Characterization of Lake Võrtsjärv. Limnologica, 28, 3-11.

Hultgren, Ch. 1988. Population dynamics and demography of Carex rostrata in relation to water level. Doctoral thesis, Uppsala University.

Jaani, A. 1973. Hüdroloogia. In Võrtsjärv (Timm, T., ed.), pp. 37-60. Valgus, Tallinn.

Jaani, A. 1990. Võrtsjärve veerežiim ja bilanss. Eesti Loodus, 11, 743-747.

Järvet, A. \& Nõges, P. 1998. Study period as compared to the long term data. In Present State and Future Fate of Lake Võrtsjärv. Results from Finnish-Estonian Joint Project in 1993-1997. (Huttula, T. \& Nõges, T., eds.), pp. 24-30. The Finnish Environment, 209 (Pirkanmaa Regional Environment Centre, Tampere).

Kalda, A. 2000. Peipsi järve ranniku taimkate. In Year-Book of the Estonian Naturalists's Society, Vol. 79, pp. 235-245. ETA Kirjastus, Tallinn.

Klinge, J. 1890. Über den Einfluss der mittleren Windrichtung auf das Verwachsen der Gewässer. Engler's bot. Jahrb., 11, 3.

Kongo, A. 1973. Võrtsjärve ümbruse füüsilis-geograafiline iseloomustus. In Võrtsjärv (Timm, T., ed.), pp. 17-25. Valgus, Tallinn.

Ksenofontova, T. 1989. General changes in the Matsalu Bay reedbeds in this century and their present quality (Estonian SSR). Aquat. Bot., 35, 111-120.

Mantai, K. E. \& Newton, M. E. 1982. Root growth in Myriophyllum: a specific plant response to nutrient availability? Aquat. Bot., 13, 45-55.

Mäemets, A. 1973. Kõrgem taimestik. In Võrtsjärv (Timm, T., ed.), pp. 77-82, 205-206. Valgus, Tallinn.

Mäemets, H. \& Mäemets, A. 2000. Commented list of macrophyte taxa of Lake Peipsi. Proc. Estonian Acad. Sci. Biol. Ecol., 49, 136-154.

Mühlen, M. von zur \& Schneider, G. 1920. Der See Wirzjerw in Livland. Archiv für die Naturkunde des Ostbaltikums, 14, 1.

Nõges, P. \& Feldmann, T. 1999. Factors controlling the distribution of aquatic macrophytes in shallow Lake Võrtsjärv. In Sustainable Lake Management. Book of Abstracts of 8th Int. Conf. Conserv. Manage. Lakes. II, p. 4.

Nõges, P., Feldmann, T. \& Nõges, T. 1999. Võrtsjärven veden laatu ja ekologia. In Võrtsjärven kunnostuksen ja suojelun yleissuunnitelma (Bilaletdin, Ä. \& Arvonen, H., eds.), pp. 22-23. Pirkanmaan Ympäristökeskus, Tampere.

Nõges, P., Feldmann, T., Haberman, J., Järvalt, A., Kangur, A., Kangur, K., Timm, H., Tuvikene, A. \& Zingel, P. 2001. Deviation of Lake Võrtsjärv from its pristine status documented 90 years ago. In Proceedings of 9 th International Conference of the Conservation and Management of Lakes, 2001, Session 5, pp. 221-224.

Nõges, T., Nõges, P., Kisand, A., Kisand, V., Tuvikene, L., Zingel, P., Põllumäe, A. \& Haberman, J. 1998. Results of empirical data analysis. In Present State and Future Fate of Lake Võrtsjärv. Results from Finnish-Estonian Joint Project in 1993-1997 (Huttula, T. \& Nõges, T., eds.), pp. 83-112. The Finnish Environment, 209 (Pirkanmaa Regional Environment Centre, Tampere).

Pastak, E. 1936. Võrtsjärve idaranniku taimkattest. Eesti Loodus, 4, 137-140.

Pokrovskaya, T. N., Mironova, N. A. \& Shil'krot, G. S. 1983. Macrophyte Lakes and Their Eutrophication. Nauka, Moskva (in Russian).

Preston, C. D. \& Croft, J. M. 1997. Aquatic Plants in Britain and Ireland. Chichester, Essex. 
Raspopov, I. M. 1985. Macrovegetation of the Large Lakes of the Northwestern of USSR. Nauka, Leningrad (in Russian).

Raukas, A. \& Mäemets, A. 1995. Võrtsjärv. In Eesti. Loodus (Raukas, A., ed.), pp. 266-273. Valgus, Tallinn.

Rintanen, T. 1996. Changes in the flora and vegetation of 113 Finnish lakes during 40 years. Ann. Bot. Fen., 33, 101-122.

Ristkok, J. \& Ruse, K. 1962. Võrtsjärve litoraali põhjafauna koosseisust ja levikust. TRÜ Toim. Zooloogia-alaseid töid II, 120.

Samuelsson, G. 1934. Die Verbreitung der höheren Wasserpflanzen in Nordeuropa (Fennoskandien und Dänemark). Uppsala.

Schmieder, K. 1998. Submerse Makrophyten der Litoralzone des Bodensees 1993 im Vergleich mit 1978 und 1967. Int. Gewässerschutzkomm. Bodensee, Bericht, 46.

Schmieder, K., Dienst, M. \& Ostendorp, W. Das Extremhochwasser 1999 am Bodensee: Auslöser eines neuen "Schilfsterbens"? (in press).

Simm, H. \& Starast, H. 1965. Võrtsjärve üldine hüdrokeemiline iseloomustus. ENSV TA Toim. Biol., 14, 57-82.

Smolders, A. J. P., Roelofs, J. G. M. \& Den Hartog, C. 1996. Possible causes for the decline of the water soldier (Stratiotes aloides L.) in Netherlands. Arch. Hydrobiol., 136, 327-342.

Sudnitsõna, D. N. 1990. Peipsi-Pihkva järve kõrgem veetaimestik. In Peipsi järve seisund I (Timm, T., ed.), pp. 87-90. Tartu.

Sviridenko, B. F. 2000. Flora and Vegetation of Reservoirs of North Kazakhstan. Pedagogical University, Omsk (in Russian).

Veber, K. 1973. Põhjasetete geoloogiast ja levikust. In Võrtsjärv (Timm, T., ed.), pp. 33-36. Valgus, Tallinn.

Wallsten, M. 1981. Changes of lakes in Uppland, Central Sweden, during 40 years. Doctoral thesis, Uppsala University.

\title{
Võrtsjärve suurtaimestiku taksonite kommenteeritud nimistu
}

\author{
Helle Mäemets
}

Nimistus on kokku võetud Võrtsjärve 114 taksonit puudutavad andmed alates Esimese maailmasõja eel kogutud materjalidest kuni 2001. aasta uuringute tulemusteni. Võrdluses Peipsi järve samalaadse nimistuga (Mäemets \& Mäemets, 2000) ilmneb kattumine enamiku liikide osas. Peipsi 128 taksoni hulgas on rohkem haruldusi ja mitmekesisem on just ajutiselt üleujutatava vööndi floora. Erinevusi nende kahe järve vahel leiab mitmete tavaliste liikide osatähtsuse muutustes viimastel aastakümnetel. Eutrofeerumisega kaasnev roostike ekspansioon, madalveeliikide väljatõrjumine ja teised taimestiku koosseisu muutused on kulgenud sarnaselt, kuid Võrtsjärves mõnevõrra kiiremini. 